\title{
Effect of Preexisting Musculoskeletal Diseases on the 1-Year Incidence of Fall-related Injuries
}

\author{
Won Kyung Lee ${ }^{1}$, Kyoung Ae Kong ${ }^{2}$, Hyesook Park ${ }^{1}$ \\ ${ }^{1}$ Department of Preventive Medicine, Ewha Womans University School of Medicine, Seoul; ${ }^{2}$ Clinical Trial Center, Ewha Womans University Medical \\ Center, Seoul, Korea
}

Objectives: People who have chronic diseases, as well as gait imbalance or psychiatric drug use, may be susceptible to injuries from falls and slips. The purpose of this study was to evaluate the effect of musculoskeletal diseases on incidental fall-related injuries among adults in Korea.

Methods: We analyzed data from the 4th Korea National Health and Nutrition Examination Survey (2007-2009), which are national data obtained by a rolling survey sampling method. The 1-year incidence of fall-related injuries was defined by health service utilization within the last year due to injury occurring after a slip and fall, and musculoskeletal diseases included osteoarthritis, rheumatoid arthritis, osteoporosis, and back pain. To evaluate the effects of preexisting musculoskeletal diseases, adults diagnosed before the last year were considered the exposed group, and adults who had never been diagnosed were the unexposed group.

Results: The weighted lifetime prevalence of musculoskeletal disease was 32540 per 100000 persons. Musculoskeletal diseases were associated with a higher risk of fall-related injury after adjustment for sex, age, residence, household income, education, occupation, visual disturbance, paralysis due to stroke, and medication for depression (odds ratio [OR], 1.41; 95\% confidence interval [Cl], 1.03 to 1.93). As the number of comorbid musculoskeletal diseases increased, the risk of fall-induced injuries increased ( $p$-value for trend $<0.001)$. In particular, patients who had any musculoskeletal condition were at much higher risk of recurrent fall-related injuries (OR, $6.20 ; 95 \% \mathrm{Cl}, 1.06$ to 36.08$)$.

Conclusions: One must take into account the risk of fall-related injuries and provide prevention strategies among adults who have musculoskeletal diseases.

Key words: Arthritis, Osteoporosis, Back pain, Accidental falls, Wounds and injuries

\section{INTRODUCTION}

Received: November 23, 2011; Accepted: March 14, 2012

Corresponding author: Hyesook Park, MD, PhD

1071 Anyangcheon-ro, Yangcheon-gu, Seoul 158-710, Korea

Tel: +82-2-2650-5756, Fax: +82-2-2652-8325

E-mail: hpark@ewha.ac.kr

This is an Open Access article distributed under the terms of the Creative Commons Attribution Non-Commercial License (http://creativecommons.org/licenses/by$\mathrm{nc} / 3.0 / /)$ which permits unrestricted non-commercial use, distribution, and reproduction in any medium, provided the original work is properly cited.
Falls are one of the major mechanisms of injury and a major cause of disability and mortality. Falls can result in fractures and mobility limitation, and are also related to many complications such as pneumonia, pressure ulcer, and thrombosis, which can lead to death among the elderly. In particular, falls are known to be a major cause of hip fracture because $95 \%$ of hip fractures result from falls [1]. Moreover, fall-related deaths constitute two-thirds of all mortality from unintentional injury 
[2]. Mortality as well as incidence of fall-related injuries has increased continuously over time. The incidence of falls among men and women aged over 80 have increased $33.6 \%$ and $28.4 \%$, respectively, each year from 1970 through 2002 in Finland [3]. In Korea, national mortality data from 1996 to 2006 have shown that falls increased to the greatest extent (24\%) among the mechanisms of injury in the elderly [4]. Additionally, hospitalization for fall-related injuries increased by $50 \%$, from 3.7 to 5.6 hundred thousand in the US [5].

Another important issue of public health is musculoskeletal disease. The prevalence of self-reported musculoskeletal conditions was 48.3 per 100 persons aged 18 and above in the US. Moreover, the cost of musculoskeletal diseases was estimated at about 849 billion dollars, $7.7 \%$ of the gross domestic product of the US [6]. In Europe, 20\% to $30 \%$ of adults have suffered from self-reported musculoskeletal disease at some point in time [7]. Korea is not an exception to the heavy burdens of musculoskeletal diseases. The prevalence of patients with arthritis was 140.0 per 1000 persons in 2001, and the cost of musculoskeletal disease was 6.89 billion dollars in $2004[8,9]$. Moreover, the burden of musculoskeletal diseases is increasing as life expectancy increases. The prevalence of arthritis in 2005 was estimated to have increased by $41 \%$ from 2001 [8]. The cost of musculoskeletal disease is predicted to increase by 8 billion dollars in 2020 [9].

The relationships between musculoskeletal diseases and fall-related injuries have been evaluated in some studies. Previous studies on musculoskeletal disease and the risk of fallrelated injuries have mainly focused on the difference in physical function tests between patients and healthy adults [10-12]. Balance impairment, muscle weakness, and pain were suggested to be related to falls. Most of those were based on retrospective data obtained from a few hospitals. It is difficult to estimate the incidence of fall-related injuries and to generalize the relationship between musculoskeletal conditions and fallrelated injuries. Therefore, the objective of this study was to evaluate the association between common musculoskeletal diseases (osteoarthritis, rheumatoid arthritis, osteoporosis, and back pain) and fall-related injuries in the general population using national data and a prospective study design.

\section{METHODS}

\section{Data and Variable Definitions}

We analyzed data from the 4th Korea National Health and
Nutrition Examination Survey (KNHANES IV), which was collected from 2007 to 2009 and evaluated the association between musculoskeletal diseases and fall-related injuries among adults in Korea. For the KNHANES IV, the Korean Ministry of Health and Welfare conducted health interview surveys on a representative sample of the community-dwelling population using a complex multistage sampling design [13]. The rolling survey sampling method was used in the KNHANES IV so that the 3-year data could be integrated.

The 1-year incidence of injuries was defined as health-care utilization due to injury within the last year. The uncertain answer "cannot remember" was excluded. Fall-related injuries were defined as those that occurred as a result of a fall or slip. Multiple fall-related injuries were confined to the fall-related injuries that occurred more than twice in the last year. In the association analysis, persons injured from falls were compared with those who experienced no injuries in the previous year.

Musculoskeletal diseases comprised osteoarthritis, rheumatoid arthritis, osteoporosis, and back pain. The survey included the current status and the chronicity of musculoskeletal diseases as well as the patients' age at the time of diagnosis of musculoskeletal diseases. The lifetime prevalence was defined as the weighted proportion of patients who have ever suffered from musculoskeletal diseases to the total population in Korea. In contrast, musculoskeletal diseases were defined conditionally as preexisting conditions diagnosed by doctors before injuries occurred, when the associations of musculoskeletal diseases and fall-related injuries were evaluated. Therefore, patients with musculoskeletal diseases were limited to the adults whose diagnosed age was at least one year less than the current age on the survey and were compared with adults who had never been diagnosed prior to the survey. Musculoskeletal diseases diagnosed within the last year were excluded because the temporal relationship between the musculoskeletal disease and fall-related injuries was not clear.

In addition to sex and age, the socioeconomic position (region, income, education status, and occupation) could be a confounding factor to injuries [14-16]. Therefore, demographic characteristics including socioeconomic position were considered in the association analysis. Occupations were divided into manual (skilled agricultural, forestry, and fishery workers; craft and related trades workers; machine operators and assemblers; and elementary workers), non-manual (managers, professionals, and related workers; clerks; service workers; and sales workers), and others (unemployed, housewife, and stu- 
dent). Quartiles of household income were substituted for individual income because the study participants included the elderly, who tend not to be economically active.

\section{Study Participants}

The number of participants who responded to the KNHANES IV from 2007 to 2009 was 24871 , and among those, the number of adults aged over 18 was 18406 . The subjects comprised 16479 individuals in the analysis for association after removing uncertain responses (61 adults who could not remember the occurrence of injuries) and missing values (1095 for injuries, 771 for injury mechanisms, and 1789 for musculoskeletal diseases).

\section{Statistical Analysis}

We integrated the data from 2007 through 2009 and estimated the lifetime prevalence of musculoskeletal diseases and 1-year incidence of fall-related injuries. Prevalence and 1-year incidence were calculated in adults aged over 18 who answered the KNHANES IV questionnaire. Survey sample weights were calculated and combined based on the sampling designs and the response rate each year. In all post-hoc analyses, sample weights were adjusted in order to make the results representative of the Korean population.

Uncertain responses and missing values were removed in the association analysis. The associations of musculoskeletal diseases and fall-related injuries were analyzed using the RaoScott test and survey logistic regression. Adjusted odds ratios (AORs) were calculated controlling for age, sex, region, education, income, and occupation. In addition, we compared them with the results that were additionally adjusted for other potential confounders (medication for depression, paralysis due to stroke, and visual acuity disturbance). Further regression analyses were performed to investigate the association of fallrelated injuries and the number of combined musculoskeletal diseases. The effects of musculoskeletal diseases on multiple fall-related injuries were also evaluated compared to the total injuries due to falls. Statistical analyses were performed using the surveyfreq and surveylogistic procedure in SAS version 9.2 (SAS Inc., Cary, NC, USA). Statistical significance was considered to be when type l error $(a)<0.05$.

\section{RESULTS}

The study subjects consisted of 18406 adults aged over 18 who answered the KNHANES IV questionnaire from 2007 to 2009. The 1-year incidence of fall-related injuries among adults was 2291 per 100000 persons per year, 33.4\% of all injuries. The persons who had ever suffered from any of the predefined musculoskeletal diseases were 6867 among 18406 adults. Considering sample weight, the lifetime prevalence of musculoskeletal diseases was 32540 per 100000 persons. Prevalence of musculoskeletal diseases was higher in the elderly than in young adults. Prevalence was 18360 per 100000 in young adults (aged 19 to 39), 35970 per 100000 in middleaged adults (aged 40 to 64), and 65980 per 100000 in elderly adults (aged 65 and over). Among the musculoskeletal diseases, back pain had the highest prevalence ( $23554 / 100000)$, followed by osteoarthritis (12 091/100 000), osteoporosis (4597/ $100000)$, and rheumatoid arthritis (2210/100 000).

Table 1 shows the general characteristics of the study participants that might act as potential confounders for the association between musculoskeletal diseases and fall-related injuries. Regarding the risk of occurrence of fall-induced injuries, no difference according to sex existed, but the elderly experienced more fall-induced injuries in the previous year than young adults. Residential district (region), education, occupation, medication for depression, and visual disturbance were associated with injuries after a fall. Fall-related injuries occurred more often among rural residents, people with low income, those with low education levels, manual workers, those taking antidepressants, and those with decreased visual acuity.

The association between preexisting musculoskeletal diseases and the incidence of fall-related injuries is shown in Table 2. People who had any musculoskeletal diseases were more likely to experience fall-related injuries. Osteoarthritis, osteoporosis, and back pain were each significantly related to the risk of fall-related injuries. Rheumatoid arthritis showed the same relationship, but it was of borderline significance $(p=0.05)$.

The incidence of fall-related injuries increased with the number of musculoskeletal diseases. The incidence of fall-related injuries in those who had one, two, and three or more musculoskeletal diseases was $2.8 \%, 3.7 \%$, and $7.7 \%$, respectively, that is, higher than in adults without musculoskeletal disease ( $p$-value for trend $<0.001$ ). Moreover, $3.0 \%, 2.9 \%$, and $3.7 \%$ of adults with short, medium, and long duration of musculoskeletal disease, respectively, experienced injuries from falls, which did not achieve a significant difference ( $p$-value for trend $=0.544$ ).

Table 3 shows the size of the risk posed by musculoskeletal disease. Adults with a musculoskeletal disease had a 1.44 times 
Table 1. General characteristics of the study participants

\begin{tabular}{|c|c|c|c|c|c|c|}
\hline & & \multicolumn{2}{|c|}{ No injury } & \multicolumn{2}{|c|}{ Fall-related injuries } & \multirow{2}{*}{$p$-value } \\
\hline & & $\mathbf{n}$ & Weighted \% & $\mathbf{n}$ & Weighted \% & \\
\hline \multicolumn{7}{|c|}{ Demographic characteristics } \\
\hline \multirow[t]{2}{*}{ Sex } & Male & 6738 & 97.53 & 169 & 2.47 & 0.18 \\
\hline & Female & 9342 & 97.88 & 230 & 2.12 & \\
\hline \multirow[t]{3}{*}{ Age $(y)^{2}$} & $19-39$ & 5368 & 98.07 & 98 & 1.93 & $<0.001$ \\
\hline & $40-64$ & 7101 & 97.77 & 164 & 2.23 & \\
\hline & $\geq 65$ & 3611 & 96.38 & 137 & 3.612 & \\
\hline \multirow[t]{2}{*}{ Region } & Urban & 11848 & 97.82 & 272 & 2.18 & 0.6 \\
\hline & Rural & 4232 & 97.23 & 127 & 2.77 & \\
\hline \multirow[t]{4}{*}{ Household income ${ }^{2}$} & Lowest & 3323 & 97.01 & 113 & 2.99 & 0.138 \\
\hline & Lower-middle & 3897 & 97.61 & 106 & 2.39 & \\
\hline & Upper-middle & 4208 & 97.92 & 84 & 2.08 & \\
\hline & Highest & 4243 & 97.92 & 88 & 2.08 & \\
\hline \multirow[t]{4}{*}{ Education $^{2}$} & Elementary school & 4650 & 96.44 & 172 & 3.56 & $<0.001$ \\
\hline & Middle school & 1792 & 98.01 & 42 & 1.99 & \\
\hline & High school & 5504 & 97.89 & 117 & 2.11 & \\
\hline & $\geq$ College & 4118 & 98.26 & 67 & 1.74 & \\
\hline \multirow[t]{3}{*}{ Occupation } & Non-manual & 4889 & 98.18 & 86 & 1.82 & 0.03 \\
\hline & Manual & 4304 & 97.34 & 121 & 2.66 & \\
\hline & Others & 6767 & 97.52 & 188 & 2.48 & \\
\hline \multicolumn{7}{|l|}{ Comorbidities } \\
\hline \multirow[t]{2}{*}{ Antidepressant } & No & 15897 & 97.74 & 386 & 2.26 & $<0.001$ \\
\hline & Yes & 183 & 93.88 & 13 & 6.12 & \\
\hline \multirow[t]{2}{*}{ Paralysis } & No & 15923 & 97.72 & 390 & 2.28 & 0.06 \\
\hline & Yes & 157 & 95.59 & 9 & 4.41 & \\
\hline \multirow[t]{2}{*}{ Visual disturbance } & No & 15199 & 97.83 & 347 & 2.17 & $<0.001$ \\
\hline & Yes & 881 & 94.58 & 52 & 5.42 & \\
\hline
\end{tabular}

${ }^{1}$ Rao-Scott chi-squared test. ${ }^{2} p$-value for trend.

higher risk of fall-related injuries than others. All four musculoskeletal diseases had a weak association (odds ratio [OR] <2) with fall-related injuries, and only back pain had a statistically significant association. Long duration of musculoskeletal disease also showed no significant relationship to fall-related injuries. However, those with three or more diseases were 3.66 times more likely to suffer from fall-related injuries than those without any disease. Significance was sustained after additionally controlling for antidepressant use, paralysis, and visual disturbance (single musculoskeletal disease OR, 1.33; 95\% confidence interval [Cl], 0.94 to 1.89; two musculoskeletal diseases $\mathrm{OR}, 1.56 ; 95 \% \mathrm{Cl}, 0.95$ to 2.54 ; three or more musculoskeletal diseases OR, 3.50; $95 \% \mathrm{Cl}, 1.45$ to 8.44; $p$-value for trend <0.01).

As for the recurrent fall-related injuries, those with musculoskeletal diseases had a much higher risk of multiple fall-related injuries within the last year $(\mathrm{OR}>6)$. Adults with medium and long duration of musculoskeletal disease experienced fall-related injuries 4.8 and 2.50 times, respectively, more often than who with short duration. However, this relationship did not show statistical significance. When the relationships between recurrent fall-related injuries and musculoskeletal diseases were evaluated, additional adjustments for antidepressant use, paralysis, and visual disturbance could not be performed because the sample size of the multiple fall-related injuries was small.

\section{DISCUSSION}

The burdens of musculoskeletal disease and fall-related injury are large in Korea. The lifetime prevalence of musculoskeletal diseases among adults aged over 18 was 32540 per 100 000 persons. The annual incidence of fall-related injuries was 
Table 2. 1-Year incidence of fall-related injuries among adults with/without musculoskeletal disease

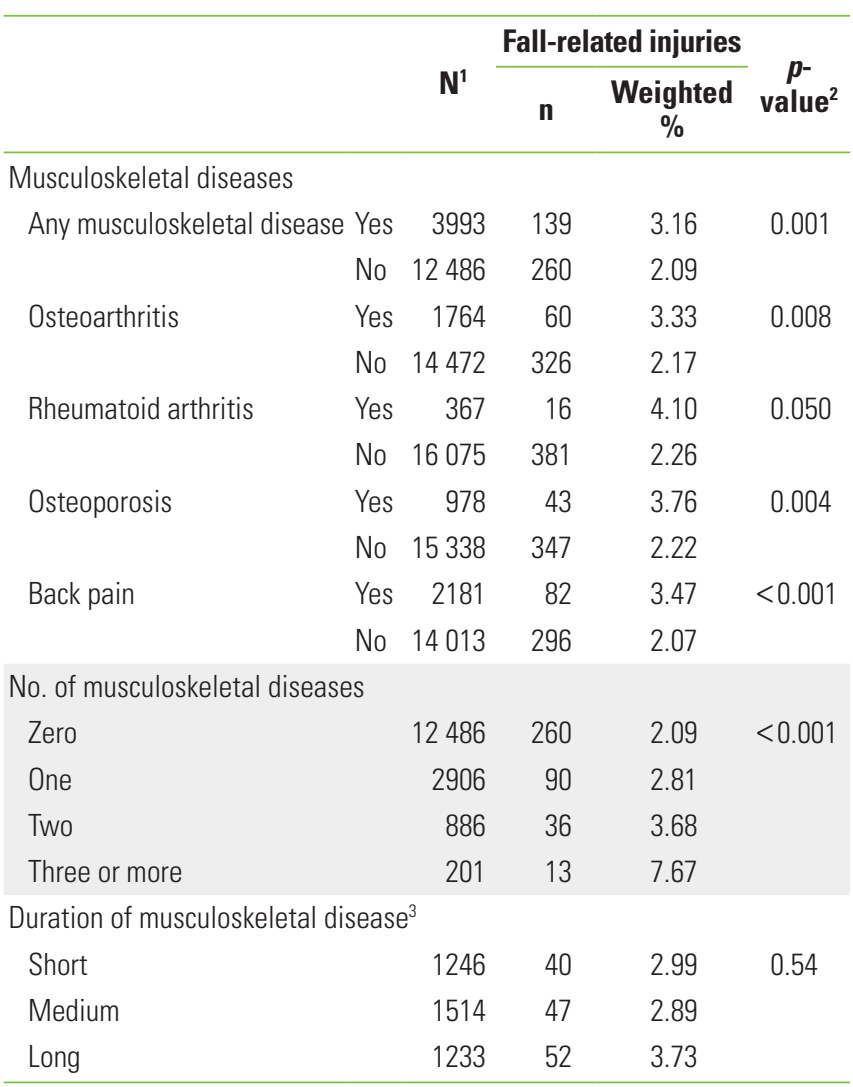

${ }^{1}$ Number of subjects. ${ }^{2}$ Rao-Scott chi-squared test. ${ }^{3}$ Short: 1 st tertile $(\leq 3 \mathrm{y})$, medium: 2nd tertile (4-10 y), long: 3rd tertile ( $\geq 11$ y).
2291 per 100000 persons. The adults with preexisting musculoskeletal diseases used more hospital services due to fall-related injuries. In particular, those who suffered from three or more diseases had a high risk of fall-related injuries. This risk was also remarkable for multiple fall-related injuries.

Falls are the major mechanism of injuries, which are a leading cause of disability and mortality among the elderly. Suggested methods of prevention of fall-related injuries include exercise training, nutritional supplementation, cognitive-behavioral approaches, environmental modification, and multidisciplinary and multifactorial interventions. Some interventions have been found effective, but others have not [17]. Therefore, researchers insist that fall prevention needs to focus on high-risk groups for efficiency and cost-effectiveness [18]. Previous studies have revealed that those who had a history of falls, muscle weakness, and/or gait imbalance are at high risk [18-21].

Musculoskeletal diseases are often accompanied by muscle weakness, gait instability, imbalance, and limitation of activities. Although adults with musculoskeletal diseases could be predisposed to fall-related injuries, the relationship has been unclear. While some previous studies have suggested relationships between osteoporosis or arthritis and falls, other studies did not find significant associations [18,22-24].

Previous reports have also suggested that patients with rheu-

Table 3. Effects of musculoskeletal diseases on the fall-related injuries among the adults

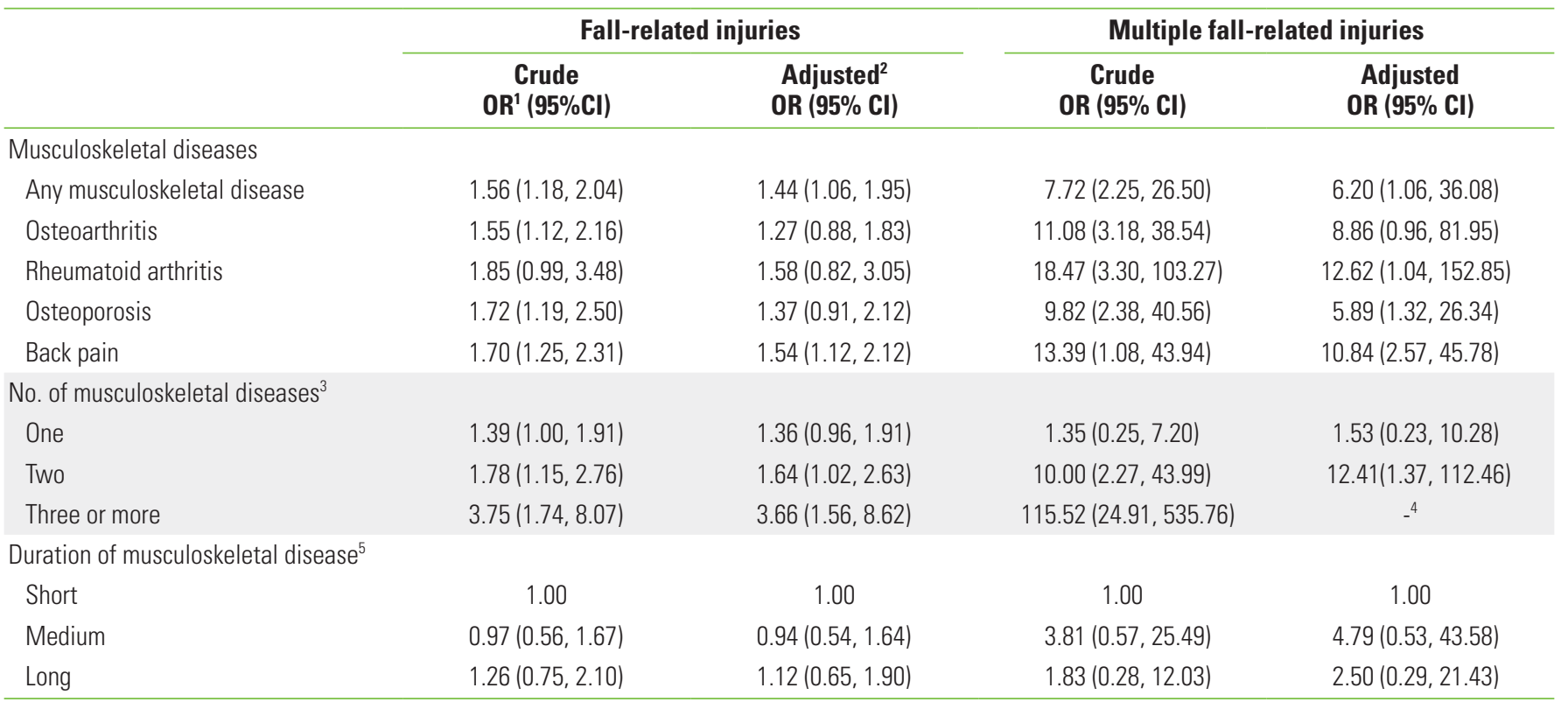

$\mathrm{OR}$, odds ratio; $\mathrm{Cl}$, confidence interval.

${ }^{1}$ Survey logistic regression. ${ }^{2}$ Adjusted for sex, age group, region, household income, education, and occupation. ${ }^{3}$ Reference: no musculoskeletal disease. ${ }^{4} 0 \mathrm{R}$ and $95 \%$ Cl were not calculated due to the small sample size. ${ }^{5}$ Short: 1 st tertile $(\leq 3 \mathrm{y})$, medium: 2 nd tertile (4-10 y), long: 3 rd tertile ( $\left.\geq 11 \mathrm{y}\right)$. 
matoid arthritis or osteoarthritis had a higher risk of falls because they had decreased balance performance and muscle strength $[2,18]$. In this study, adults with rheumatoid arthritis or osteoarthritis were more likely to be injured from falls. These associations were weak $(O R<2)$ and not significant. The previous reports on the risk of fall-related injuries and arthritis showed inconsistent results. A case-control study in the United Kingdom showed results similar to ours (OR, 1.50; $95 \% \mathrm{Cl}, 0.95$ to 2.46) [25]. In contrast, a cohort study in the United States revealed a significant and strong effect of preexisting rheumatoid arthritis on fall injury (OR, 2.00; $95 \% \mathrm{Cl}, 1.28$ to 3.13$)$ [12].

Osteoporosis is known to increase vulnerability to fractures after falls. Recently, some researchers have suggested that adults with osteoporosis were susceptible to not only fractures but also falls themselves [26]. Earlier studies have used physical performance tests to assess the likelihood of fall-related injuries. They showed that muscle strength and balance performance decreased $18 \%$ and $11 \%$, respectively, in women with osteoporosis $[27,28]$. Posture change such as kyphosis in patients with osteoporosis was also proposed as a risk factor for falls. However, a recent study implied that such patients did not have a decreased ability to avoid obstacles and had good balance confidence [12]. Our study is consistent with these concepts. We could not find a significant relationship between osteoporosis and fall-related injuries (AOR, 1.25; 95\% Cl, 0.82 to 1.92). However, the evidence is not sufficient to determine the effect of osteoporosis on fall-induced injuries. Therefore, further research is needed to establish the relationship and the mechanism.

Back pain is a symptom of various diseases such as lumbar spondylosis, herniated intervertebral disc, osteoarthritis, and spinal stenosis. Recent studies have revealed that not only lumbar spondylosis and spinal stenosis, but also backache itself, was associated with recurrent falls $[11,29]$. Adults with back pain could experience repeated falls through static or gait imbalance [30]. The association between back pain and fall-related injuries was weak (AOR, 1.46) in our study. It was similar to the results of a study conducted in Japan (AOR, 1.34), although making further comparisons is impossible due to lack of detailed information [11].

The overall effects of musculoskeletal diseases on fall-related injuries were modest in this study. However, a much stronger effect was found for recurrent fall-related injuries. Falls are multifactorial events involving individual and environmental conditions, and recurrent falls are affected by individual fac- tors much more than single falls [11]. Previous studies have suggested that individual risk factors did not have a strong association with a single fall but did have strong predictive power for recurrent falls [23].

Musculoskeletal diseases occur mainly in the elderly, and the mechanisms are related to one another [31]. Therefore, the elderly could suffer from multiple musculoskeletal diseases simultaneously. Adults with more than two musculoskeletal diseases accounted for $22 \%$ of the total patients in Korea. Those who had three or more musculoskeletal diseases had three times the risk of fall injury seen in those without disease. These findings imply that osteoarthritis, rheumatoid arthritis, osteoporosis, and back pain have multiplicative interactive effects on the risk of fall-related injuries. Additionally, although the relationship between the duration of musculoskeletal diseases and multiple fall-related injuries could not reach statistical significance, the effect size was more than moderate for the disease lasting 4 years or longer (AOR, 4.79; 2.50 for medium and long duration). It was possible that the risk of fall-related injuries varies based on the duration of musculoskeletal diseases, which could be related to disease progression and physical performance. Therefore, patients with musculoskeletal diseases for a long duration who have had a history of fall should be strategic targets for a prevention program.

The risk of fall-related injuries was estimated relative to no injury. When the risk was calculated compared to the other types of injuries, the effect sizes were somewhat lower. Nevertheless, most of them still showed significant relationships. Adults with any musculoskeletal disease suffered from multiple fall-related injuries 4.46 times more often than other types of injuries. The AORs of osteoarthritis, rheumatoid arthritis, osteoporosis, and back pain were $6.63(95 \% \mathrm{Cl}, 1.24$ to 35.47$)$, 9.91 (95\% Cl, 1.14 to 86.28$), 5.11$ ( $95 \% \mathrm{Cl}, 1.16$ to 22.46 ), and 7.66 (95\% Cl, 1.73 to 33.85$)$, respectively. In addition, when patients had two musculoskeletal diseases, the risk to multiple fall-related injuries was 9.19 ( $95 \% \mathrm{Cl}, 0.86$ to 98.12 ).

A strength of this study was the ability to estimate the association between musculoskeletal diseases and fall-related injuries using representative data, which make the results generalizable. It also could satisfy the temporal relationship because musculoskeletal diseases were defined as conditions diagnosed before the past year so that the diagnosis preceded the fall-related injuries. Therefore, our analysis implied that musculoskeletal diseases could have a causal relationship with fall-related injuries. In addition to the various effects of muscu- 
loskeletal diseases on fall-related injuries, the different effects on overall and recurrent fall-related injuries could be clarified in this study.

This study had some limitations. First, study participants were confined to the community-dwelling population who visited a hospital after a fall. Although a possibility of selection bias existed, which excluded mildly and severely injured patients, our results could be meaningfully interpreted for patients with injuries of moderate severity. Second, information bias was possible because the KNHANES was based on a questionnaire rather than medical records. The risk of information bias, however, was modest because bias is less likely to be directional when analyzing secondary data. Third, we could not assess the functional characteristics related to fall-related injuries such as balance, muscle strength, and obstacle avoidance. Forth, residual confounding effects of age could be possible because age groups were used in the analysis. However, when the data were analyzed by age instead of the age group, the change was not a significant decrease in effect size but a widening of the confidence interval.

In conclusion, this study suggested that musculoskeletal diseases could be predisposing factors for fall-related injuries among the adult population in Korea. In particular, the relationship became much stronger for recurrent fall-related injuries. Adults with concurrent musculoskeletal diseases had a higher risk of fall-related injuries than those with a single disease. People with multiple musculoskeletal diseases could serve as a target group for prevention strategies. Effective screening and intervention programs for patients with musculoskeletal disease could prevent recurrent fall-related injuries to improve health and reduce hospital visits.

\section{CONFLICT OF INTEREST}

The authors have no conflicts of interest with the material presented in this paper.

\section{REFERENCES}

1. Barrett-Connor E, Weiss TW, McHorney CA, Miller PD, Siris ES. Predictors of falls among postmenopausal women: results from the National Osteoporosis Risk Assessment (NORA). Osteoporos Int 2009;20(5):715-722.

2. Hayashibara M, Hagino H, Katagiri H, Okano T, Okada J, Teshima $R$. Incidence and risk factors of falling in ambulatory pa- tients with rheumatoid arthritis: a prospective 1-year study. Osteoporos Int 2010;21(11):1825-1833.

3. Kannus P, Niemi S, Palvanen M, Parkkari J. Rising incidence of fall-induced injuries among elderly adults. J Public Health 2005; 13(4):212-215.

4. Hong J, Lee WK, Park H. Change in causes of injury-related deaths in South Korea, 1996-2006. J Epidemiol 2011;21(6): 500-506.

5. Hartholt KA, Stevens JA, Polinder S, van der Cammen TJ, Patka $P$. Increase in fall-related hospitalizations in the United States, 2001-2008. J Trauma 2011;71(1):255-258.

6. The Burden of Musculoskeletal Diseases in the United States. The burden of musculoskeletal diseases in the United States [cited 2012 Sep 17]. Available from: http://www.boneandjointburden.org.

7. Slim ZN, Chaaya M, Habib RR, Arayssi T, Uthman I. High burden of musculoskeletal conditions: a problem that has only recently come to recognition. Chronic IIIn 2011;7(4):311-320.

8. Hur NW, Choi CB, Uhm WS, Bae SC. The prevalence and trend of arthritis in Korea: results from Korea National Health and Nutrition Examination Surveys. J Rheum Dis 2008;15(1):11-26 (Korean).

9. Oh IH, Yoon SJ, Seo HY, Kim EJ, Kim YA. The economic burden of musculoskeletal disease in Korea: a cross sectional study. BMC Musculoskelet Disord 2011;12:157.

10. Clyburn TA, Heydemann JA. Fall prevention in the elderly: analysis and comprehensive review of methods used in the hospital and in the home. J Am Acad Orthop Surg 2011;19(7):402409.

11. Muraki S, Akune T, Oka H, En-Yo Y, Yoshida M, Nakamura K, et al. Prevalence of falls and the association with knee osteoarthritis and lumbar spondylosis as well as knee and lower back pain in Japanese men and women. Arthritis Care Res (Hoboken) 2011;63(10):1425-1431.

12. Smulders E, van Lankveld W, Laan R, Duysens J, Weerdesteyn V. Does osteoporosis predispose falls? A study on obstacle avoidance and balance confidence. BMC Musculoskelet Disord 2011; $12: 1$.

13. Korean Ministry of Health and Welfare. Korea National Health and Nutrition Examination Survey [cited 2012 Sep 17]. Available from: http://knhanes.cdc.go.kr.

14. Cubbin C, LeClere FB, Smith GS. Socioeconomic status and the occurrence of fatal and nonfatal injury in the United States. Am J Public Health 2000;90(1):70-77.

15. Burrows S, Auger N, Gamache P, Hamel D. Individual and area 
socioeconomic inequalities in cause-specific unintentional injury mortality: 11-year follow-up study of 2.7 million Canadians. Accid Anal Prev 2012;45:99-106.

16. Borrell C, Plasencia A, Huisman M, Costa G, Kunst A, Andersen $O$, et al. Education level inequalities and transportation injury mortality in the middle aged and elderly in European settings. Inj Prev 2005;11(3):138-142.

17. Gillespie LD, Gillespie WJ, Robertson MC, Lamb SE, Cumming $\mathrm{RG}$, Rowe $\mathrm{BH}$. Interventions for preventing falls in elderly people. Cochrane Database Syst Rev 2003;(4):CD000340.

18. Bongue B, Dupre C, Beauchet O, Rossat A, Fantino B, Colvez A. A screening tool with five risk factors was developed for fallrisk prediction in community-dwelling elderly. J Clin Epidemiol 2011;64(10):1152-1160.

19. Ganz DA, Bao Y, Shekelle PG, Rubenstein LZ. Will my patient fall? JAMA 2007;297(1):77-86.

20. Stevens JA, Olson $S$. Reducing falls and resulting hip fractures among older women. MMWR Recomm Rep 2000;49(RR-2):312.

21. Muir SW, Berg K, Chesworth BM, Klar N, Speechley M. Modifiable risk factors identify people who transition from non-fallers to fallers in community-dwelling older adults: a prospective study. Physiother Can 2010;62(4):358-367.

22. Roe B, Howell F, Riniotis K, Beech R, Crome P, Ong BN. Older people and falls: health status, quality of life, lifestyle, care networks, prevention and views on service use following a recent fall. J Clin Nurs 2009;18(16):2261-2272.

23. Nevitt MC, Cummings SR, Kidd S, Black D. Risk factors for recurrent nonsyncopal falls. A prospective study. JAMA 1989; 261(18):2663-2668.
24. Vu T, Finch CF, Day L. Patterns of comorbidity in communitydwelling older people hospitalised for fall-related injury: a cluster analysis. BMC Geriatr 2011;11:45.

25. Kaz Kaz H, Johnson D, Kerry S, Chinappen U, Tweed K, Patel S. Fall-related risk factors and osteoporosis in women with rheumatoid arthritis. Rheumatology (Oxford) 2004;43(10):12671271.

26. Cummings-Vaughn LA, Gammack JK. Falls, osteoporosis, and hip fractures. Med Clin North Am 2011;95(3):495-506.

27. Liu-Ambrose T, Eng JJ, Khan KM, Carter ND, McKay HA. Older women with osteoporosis have increased postural sway and weaker quadriceps strength than counterparts with normal bone mass: overlooked determinants of fracture risk? J Gerontol A Biol Sci Med Sci 2003;58(9):M862-M866.

28. Arnold CM, Busch AJ, Schachter CL, Harrison L, Olszynski W. The relationship of intrinsic fall risk factors to a recent history of falling in older women with osteoporosis. J Orthop Sports Phys Ther 2005;35(7):452-460.

29. Kim HJ, Chun HJ, Han CD, Moon SH, Kang KT, Kim HS, et al. The risk assessment of a fall in patients with lumbar spinal stenosis. Spine (Phila Pa 1976) 2011;36(9):E588-E592.

30. Liu-Ambrose T, Eng JJ, Khan KM, Mallinson A, Carter ND, McKay HA. The influence of back pain on balance and functional mobility in 65- to 75-year-old women with osteoporosis. Osteoporos Int 2002;13(11):868-873.

31. Kim SY, Schneeweiss S, Liu J, Daniel GW, Chang CL, Garneau K, et al. Risk of osteoporotic fracture in a large population-based cohort of patients with rheumatoid arthritis. Arthritis Res Ther 2010;12(4):R154. 